\title{
Study and Design on an Improved AODVjr Routing Algorithm
}

\author{
Hu Zhiqiang ${ }^{1,2, a *}$, Chen Yue ${ }^{1, b}$ and Zhang Jianhua ${ }^{1, c}$ \\ 1 Jiangsu Key Laboratory of Large Engineer Equipment Detection and Control \\ Xuzhou Institute of technology, Xuzhou 221111, China \\ ${ }^{2}$ School of Information and Electrical Engineering \\ China University of Mining and Technology, Xuzhou 221000 , China \\ ahuzhiqiangxn@126.com, ${ }^{b} 76344835 @ q q . c o m,{ }^{c} 26522808 @ q q . c o m$
}

\section{Keywords: Zigbee; AODVjr; Clustering; Energy consumption}

Abstract: Aiming AODVjr existing zigbee routing algorithm used in the network node energy consumption leads to low energy loss problems quickly, this article from the node to start binding energy clustering mechanism AODVjr propose an improved algorithm by the route selection process according to the current node energy to avoid its lower energy node clustering, and high energy to strike the optimal routing path node. Simulation results show that the algorithm can effectively reduce the total energy consumption and the number of deaths zigbee network nodes and prolong the life cycle of zigbee network.

\section{Introduction}

Nowadays, internet of things is used extensively on the base of wireless sensor network as the great support of country on the internet of things. Zigbee WSN is used widely in military, electricity, smart home etc. and becomes the mainstream of WSN because of its advantage of low energy consumption and high stability[1-4].

Nevertheless, with the further use of the Zigbee technology, especially in use of the hostile environment like military reconnaissance and power line monitoring, the survive energy of Zigbee network is challenged greatly. Network energy consumption is one of the important factors of the network survive capacity. Normally, the lower total energy consumption, the longer network life cycle. In Zigbee WSN, network routing is an important segment that effects the total energy consumption, and it will consume most of network energy in order to ensuring network stable network link. Therefore, how to balance network energy consumption and network stability is a great issue in recent network routing design[5]. AODVjr routing algorithm is used widely in Zigbee network application with low energy consumption. However, the traditional AODVjr routing algorithm use the group of flood during routing selection, and there are a lot of routing request packets flooding problem during communication lead to Zigbee node's energy quickly consuming , result in sensor node decreased greatly and reduced the life cycle of the whole Zigbee internet[6]. Therefore, how to improve the AODVjr algorithm performance and reduce the energy consumption of network routing becomes an urgent issue need to be addressed to meet the complicate and high performance internet environment[7-8].

Under this background, this paper raises an improved ODVjr algorithm in considering of the current node energy. This algorithm will avoid the low energy node when select routing, reduce Zigbee network energy consumption and number of dead node to lengthen Zigbee network life cycle by adding node energy recognition according to the energy cluster mechanism. 


\section{AODV protocol and AODVjr algorithm}

AODV protocol is an On-demand plane vector protocol used in WSN (Wireless Sensor Network). It is a dynamic routing protocol. Compared with the traditional static routing protocol, AODV protocol is no need to update internet routing list by transmit information periodically [9]. It is only need to be requested to start searching internet routing when data need to be transmitted. This way avoid the network computing bandwidth and energy consumption from periodical routing, and well suit to the WSN with a limited energy and high restrict of energy consumption. In AODV protocol, the routing loop is avoided in repairing the broken link by bringing in data package serial number and HELLO message [10]. Meantime, the link status is notified to sensor node timely, and AODV protocol high communication efficiency is ensured even though the network size and node number are enlarged [11].

AODVjr protocol is a simplified AODV protocol which is widely used in Zigbee network routing algorithm protocol, it will achieve less energy consumption and easy to realize. Moreover, the performance of AODVjr protocol is very close to AODV protocol, and more suitable to WSN with a limited energy and high strict of energy consumption. AODVjr protocol will make the most ensure of AODV protocol and save WSN energy consumption.

Working principle of AODVjr protocol is shown as below Fig.1.The life cycle is controlled by updating only when receiving valid routing data package. When routing is broken, the source node will stop receiving data after waiting for a certain period of time and there is no data was transmitted from the node and the next routing request is made. End-to-end communication strategy is used in AODVjr protocol; therefore HELLO message mechanism and mistake report mechanism are omitted and will not affect the routing stability. In this way, network cost reduced greatly. However, in AODVjr protocol, low energy nodes are dying early and affect the network life cycle cause of no consider of current energy in current node during routing request and routing selection[12].

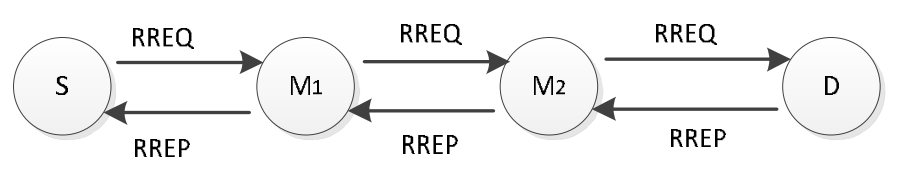

Fig. 1 Working principle of AODVjr protocol

\section{Improved AODVjr algorithm}

Through analysis of AODV protocol and AODVjr routing algorithm, AODVjr algorithm takes the whole network node as the request object when sending data packages, and didn't take account of low energy node was joint up routing link lead to low energy node consumption and die quickly, and therefore affect the whole Zigbee network life cycle[13]. Based on this issue, this paper is start from the Zigbee network energy consumption and life cycle; use the view of Cluster-Tree algorithm firstly, evaluate the direction of RREP request package, judge the RREP probable direction, and bring in the current node energy parameter, to measure the useable node energy in network, cluster it to high, middle low level according to the energy consumption. Give priority to high energy node when selecting new routing and then middle energy node and the last low energy node. By this way, low energy node join up will be reduced and give way to high energy node; therefore the node energy consumption is balanced in network, reduced the whole network energy consumption due to the low energy node in the process of maintain normal communication need to consume more energy, and lengthen the Zigbee network life cycle greatly. 
Based on above improving plan, this paper adds the energy mark ener in RREQ data package to mark the current energy consumption by designing the AODVjr algorithm. ener=- 1 means low energy status, ener $=0$ means middle energy status, ener $=1$ means high energy status. And low energy status stands for energy is lower than $20 \%$ of the total; middle level stands for energy is lower than $80 \%$ and higher than $20 \%$; and high level stands for energy higher than $80 \%$. The improved AODVjr algorithm implements steps as below:

1) When each of the nodes receiving RREQ data packages from the previous node, record the previous node energy status and add it to energy status cluster, and judge whether this node is the final node. If yes, reply RREQ; if not judge self-energy status first. If self-energy is in a low level, transfer to 2), otherwise update ener value of RREQ and then transfer;

2) Detect the nearby node energy status in low energy cluster list. If the nearby node is in a low energy status, transfer it. Otherwise comes to 3);

3) Detect whether the hop count is beyond the limitation. If the depth is beyond the limitation by tree-form routing calculation, discard the RREQ data package. Otherwise comes to 4);

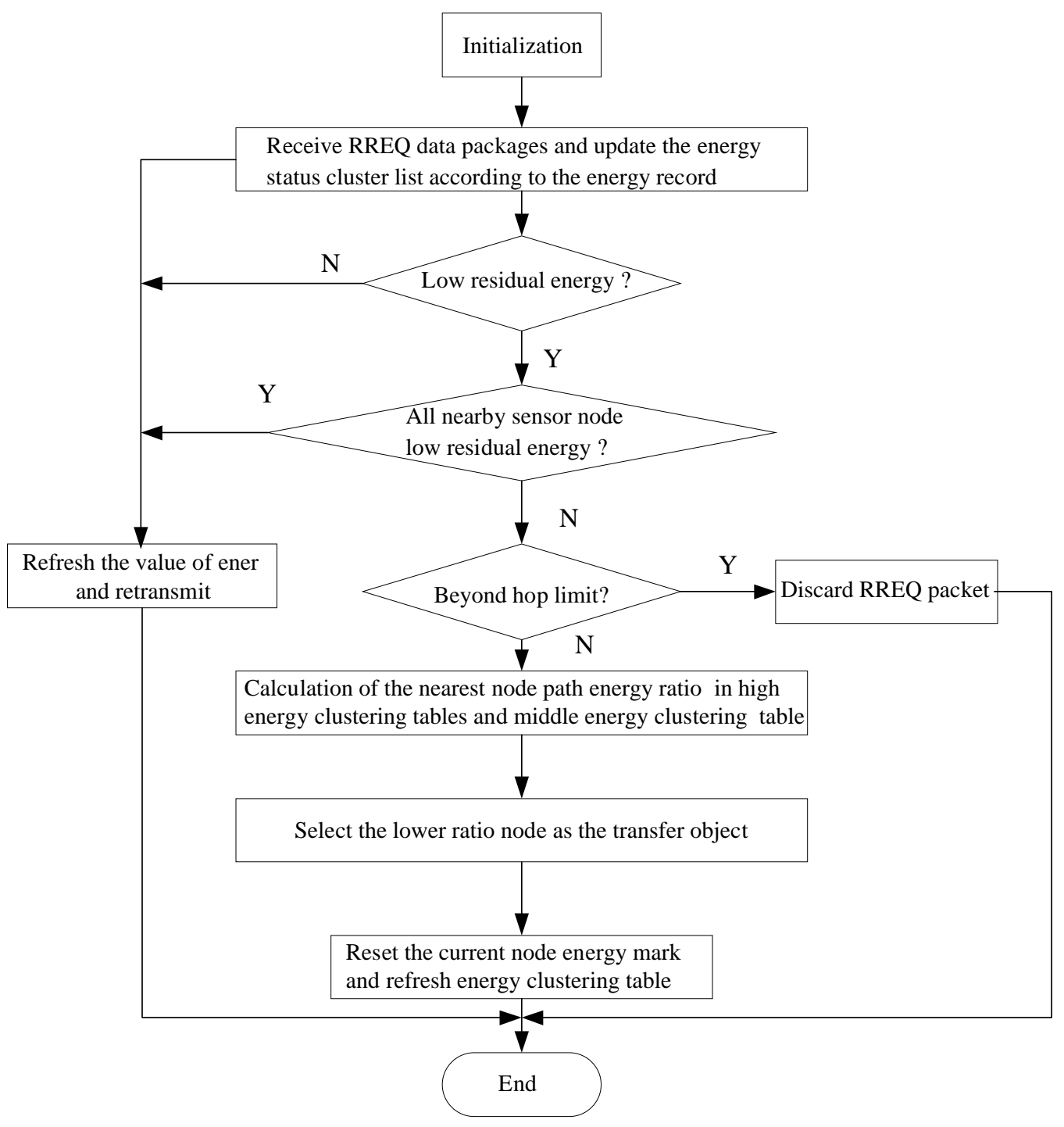

Fig. 2 Flow chart of the node process RREQ data package in improved AODVjr algorithm 
4) Find out the nearest high energy node in high energy cluster list and the nearest middle energy node in middle energy cluster list, and calculate the path energy ratio. Select the lower ratio node as the transfer object and transfer the data. The path energy ratio will be calculated as below formula (1):

$$
P=N / E
$$

In this formula, $\mathrm{P}$ is the path energy ratio, $\mathrm{N}$ is path hop count, and $\mathrm{E}$ is node energy value.

5) Reset the current node energy mark, and update node energy information in energy cluster list according to the RREQ data package received.

Above is the RREQ data package processing in the whole middle node. Finally, if the source node received the RREP group from destination node, transmit data according to the path found by node, and update self-energy status in energy cluster. Fig.2 is the whole algorithm execution flow chart.

\section{Result and discussion}

To verify the improved AODVjr algorithm performance in this paper, the NS2 network simulation experiment is done. The simulation environment is : network range $300 \mathrm{~m} \times 300 \mathrm{~m}$, communication radius is $15 \mathrm{~m}$, data package length is 64 bit. CBR is used as the data information source, network node is 200 , and network parameter is $\mathrm{Cm}=5, \mathrm{Rm}=4, \mathrm{Lm}=6$. The primary energy of each node is $1500 \mathrm{~J}$. The source node of data group and aim node is selected randomly. By analyzing the whole network energy consumption, network node die number, success rate of data package transmitting and compare with common AODVjr algorithm, a,b,c in Figure 3 are achieved.

From a in Figure 3, the network energy consumption of improved AODVjr algorithm is lower than the common AODVjr algorithm. The improved AODVjr algorithm has a network energy consumption of $7.8 \mathrm{Kj}$ while the common AODVjr algorithm has a network energy consumption of $11.5 \mathrm{Kj}$ when network flow is 2.0 and will be obviously showed while network whole flow is increased. The energy consumption can reach $6 \mathrm{kj}$ when network flow is 5.0. We can see from figure $\mathrm{b}$, the improved AODVjr algorithm raised in this paper has a lower number of dead nodes than the common AODVjr algorithm with the increase of network transmission. This is more obviously when the network total flow exceed 3.0. The improved AODVjr algorithm has dead node of 7, however the common AODVjr algorithm has dead node more than 10 and this gap will enlarge while the network flow is increased. The common AODVjr algorithm has 15 more dead nodes than the improved one when network flow reaches 5.0. From figure $\mathrm{c}$ we can see the data package transferring success rate of the improved AODVjr algorithm is higher than the common algorithm and this gap will show more obviously when network flow exceeds 3.0. The improved AODVjr algorithm has a success rate of $75 \%$ while the common algorithm can only reach nearly $60 \%$. This gap is obviously with the increase of the network flow, and the gap is $20 \%$ when the network flow reach 5.0. The comprehensive comparison shows that the improved AODVjr algorithm raised in this paper has a higher performance, and it is lengthen the whole network life cycle and improved the network stability greatly when it be used in Zigbee network. 


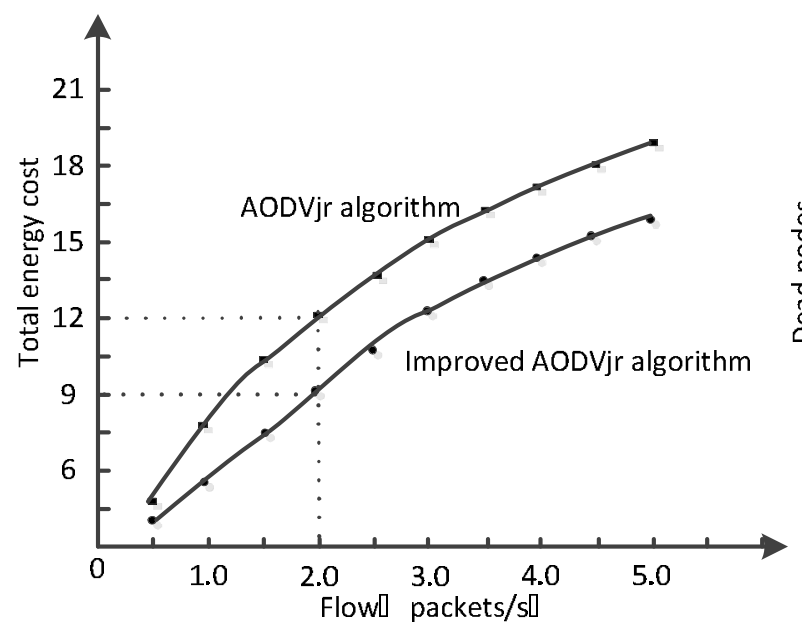

a Comparison of the Network total energy cost

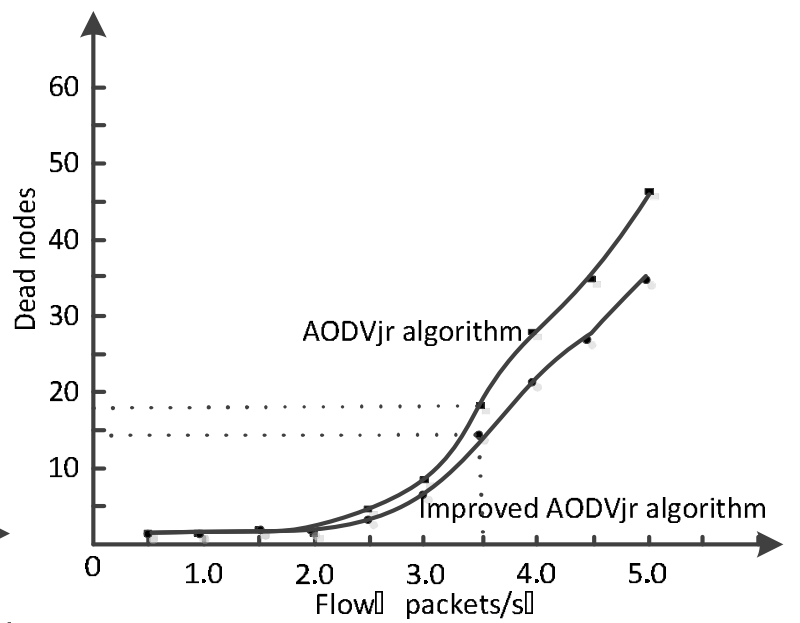

b Comparison of the dead nodes

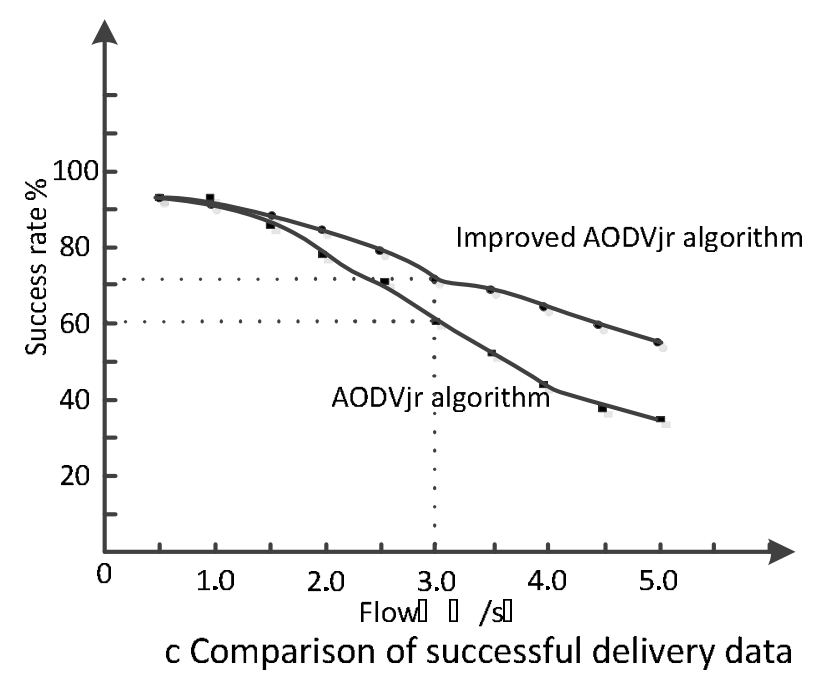

Fig. 3 NS2 Simulation Experiment result

\section{Conclusion}

An improved AODVjr algorithm is raised in this paper by starting with the node energy and cluster mechanism. Through clustering the node during node selection and avoid the low energy node, and selecting the high energy and best path, prevent the AODVjr algorithm flood routing. In the last, the NS2 simulation experiment is done and the result showed this algorithm can reduce the Zigbee network energy consumption and the number of node die, lengthen Zigbee network life cycle. This has a great significance to the WSN technology development and application.

\section{ACKNOWLEDGMENT}

This work is supported by the University Natural Science Research Project of Jiangsu Province, China (No.13KJD510008), and the Large Engineering Equipment Detection and Controlling Key Construction Laboratory Opening Project of Jiangsu Province, China(No.JSKLEDC201311).

\section{REFERENCES}

[1]Han Dawei. Study of Energy Saving Based on The Network Layer AODVjr Zigbee Routing Algorithm[D].Hangzhou Dianzi University, 2011.

[2]Liu Zhiling. Research and Improvement of AODVjr Protocol Based on ZigBee Wireless Sensor 
Network[D]. South China University of Technology, 2013.

[3]Xiechuan. Research of AODVjr Algorithm Based on ZigBee[J]. Computer Engineering,2011;10(37):87-89.

[4] Design of home wireless network using routing algorithm of Tree and AODVjr-PB[J]. Journal of Chongqing University of Posts and Telecommunications(Natural Science), 2011,03:343-348.

[5]Shen Da. Research on Data Monitoring System of Oil Field Based on AODVjr Routing Protocol and Zigbee Technology[D]. Yanshan University,2014

[6]Zou Guoxia, Tang Jianqing. Improvement of AODVjr routing algorithm in wireless sensor network[J]. Computer Era,2013,01:9-11.

[7]Qian Zhihong, Zhu Shuang, Wang Xue. An Cluster-Based Zigbee Routing Algorithm for Network Energy Optimization[J]. Chinese Journal of Computers , 2013,03:485-493.

[8]Fan Qiu, Jianming Wang, Jing Leng, Xiangui Xiao . Design and Implementation of a Wireless Personal Area Network Based on AODVjr Routing.ICWMMN 2006 Proceedings,2006:424-427.

[9] Yingqi Niu, Hongxia Bie. An improved AODVjr algorithm for extending network lifetime. Proceedings of IC-NIDC,2012:18-21.

[10]Wang Fengzhu. Research on Reliable Routing Protocol for Wireless Sensor Networks[D]. North China Electric Power University, 2013.

[11]Shi Yongsheng. Improvement of AODVjr routing algorithm in wireless sensor network[J].Electronic Technology \& Software Engineering, 2013,22:63-64.

[12]Luo Wubin, Luo Darong. Research on the Zigbee Routing Protocal[J]. Computer Engineering \& Science, 2009,06:12-14.

[13] Zhou Xiaowu, Xu Du, Jiang Yongping, Zhou Yancan. Analysis and Improvement for Basic Zigbee Wireless Networks Route[J]. Computer Knowledge and Technology, 2009,33:9242-9245. 